\title{
The dynamics of HIV infection model with logistic growth and infected cells in eclipse phase
}

\author{
Sanaa Harroudi, a and Karam Allali, ${ }^{\text {b }}$ \\ Department of Mathematics, FSTM, Laboratory of Mathematics \& Applications , University Hassan II-Casablanca, PO \\ Box146, Mohammedia, Morocco
}

\begin{abstract}
In this paper, we study a mathematical model of human immunodeficiency virus dynamics with logistic growth and infected cells in eclipse phase. This model describes the interactions between uninfected $\mathrm{CD}^{+} \mathrm{T}$ cells, infected $\mathrm{CD} 4^{+} \mathrm{T}$ cells in latent stage, productively infected $\mathrm{CD} 4^{+} \mathrm{T}$ cells and free virus. The positivity and boundedness of solutions for non negative initial data are proved. The stability of disease-free equilibrium and endemic equilibrium are rigorously established. Numerical simulations are also provided to give a more complete representation of the system dynamics.
\end{abstract}

Keywords: HIV infection, Logistic growth, Stability, Viral dynamics.

\section{Introduction}

Human immunodeficiency virus (HIV) is a pathogen which causes the well known acquired immunodeficiency syndrome (AIDS). HIV continues to be a major global public health issue, having claimed more than 35 million lives so far; in 2016 more than 1 million people died from HIV-related causes globally [1]. Without treatment of the HIV infection, HIV advances in stages getting worse over time [2]. However, the most powerful antiretrovirals can not completely eliminate the virus because it remains dormant in some cells [3]. In the last decades, many mathematical models have proved to be useful for describing and understanding the dynamics of HIV infection by considering three classes of $\mathrm{CD}^{+}{ }^{+} \mathrm{T}$ cells: uninfected cells, infected cells in latent phase and productively infected cells [4-6]. In recent years, much works extend the basic model by including a logistic growth term that describes the growth rate of healthy $\mathrm{CD} 4^{+} \mathrm{T}$ cells $[7,8]$, since the proliferation rate of T-cells is density-dependent with the rate of proliferation slowing as the T-cell count gets high [7]. In this study, we extend the recent work [9] by considering a logistic growth rate instead of constant one and the model that we consider is the following

$$
\left\{\begin{array}{l}
\dot{x}=r x(t)\left(1-\frac{T(t)}{T_{m}}\right)-d_{1} x(t)-\frac{k_{1} x(t) v(t)}{x(t)+v(t)} \\
\dot{y}=\frac{k_{1} x(t) v(t)}{x(t)+v(t)}-\left(d_{2}+k_{2}\right) y(t) \\
\dot{s}=k_{2} y(t)-d_{3} s(t) \\
\dot{v}=a s(t)-d_{4} v(t)
\end{array}\right.
$$

With

$$
T(t)=x(t)+y(t)+s(t)
$$

\footnotetext{
a e-mail: sanaa.harroudi@gmail.com

b e-mail: allali@hotmail.com
}

The initial data are

$$
\begin{gathered}
x(0)=x_{0} \geq 0, y(0)=y_{0} \geq 0, s(0)=s_{0} \geq 0, v(0)=v_{0} \geq 0, \\
T_{m} \geq T_{0}=x_{0}+y_{0}+s_{0}>0 .
\end{gathered}
$$

In this model, $x(t), y(t), s(t), v(t)$ represent the concentration of uninfected $\mathrm{CD}^{+} \mathrm{T}$ cells, infected $\mathrm{CD} 4^{+} \mathrm{T}$ cells in latent stage, productively infected $\mathrm{CD}^{+}{ }^{+} \mathrm{T}$ cells and free virus (HIV), respectively. The uninfected CD4 ${ }^{+}$ T cells grow at a rate $r$, die at a rate $d_{1}$ and become infected by free virus a rate $k_{1} . T_{m}$ is the carrying capacity of the T-cell population. Infected $\mathrm{CD}^{+}{ }^{+} \mathrm{T}$ cells in latent stage are produced at a rate $k_{1}$, die at a rate $d_{2}$ and become productively infected cells at a rate $k_{2}$. Productively infected $\mathrm{CD} 4^{+} \mathrm{T}$ cells are produced at a rate $k_{2}$ and die at a rate $d_{3}$. Free virus (HIV) is produced from infected cells at a rate $a$ and die a rate $d_{4}$.

The paper is organized as follows. The next section is devoted to the analysis of the model which contain the existence, positivity, boundedness of solutions and the stability analysis. Section 3, deals with some numerical simulations of the model. Finally, we conclude in the last section.

\section{Analysis of the model}

\subsection{Steady states}

There exist two steady states of the studied model: The infection-free equilibrium $E_{f}=\left(\frac{T_{m}\left(r-d_{1}\right)}{r}, 0,0,0\right)$ which represent the disease free equilibrium and correspond to the maximal level of healthy CD4 ${ }^{+} \mathrm{T}$ cells. $E^{*}=\left(x^{*}, y^{*}, s^{*}, v^{*}\right)$ is a state of persistent, chronic HIV infection. Explicitly, $E^{*}$ requires

$$
x^{*}=\frac{T_{m} d_{3} d_{4}\left(d_{2}+k_{2}\right)\left(R^{*}-R_{0}\right)}{r\left(d_{4}\left(d_{3}+k_{2}\right)\left(R_{0}-1\right)+a k_{2}\right)}
$$




$$
\begin{aligned}
& y^{*}=\frac{T_{m} d_{3}^{2} d_{4}^{2}\left(d_{2}+k_{2}\right)\left(R_{0}-1\right)\left(R^{*}-R_{0}\right)}{a r k_{2}\left(d_{4}\left(d_{3}+k_{2}\right)\left(R_{0}-1\right)+a k_{2}\right)}, \\
& s^{*}=\frac{T_{m} d_{3} d_{4}^{2}\left(d_{2}+k_{2}\right)\left(R_{0}-1\right)\left(R^{*}-R_{0}\right)}{\operatorname{ar}\left(d_{4}\left(d_{3}+k_{2}\right)\left(R_{0}-1\right)+a k_{2}\right)}, \\
& v^{*}=\frac{T_{m} d_{3} d_{4}\left(d_{2}+k_{2}\right)\left(R_{0}-1\right)\left(R^{*}-R_{0}\right)}{r\left(d_{4}\left(d_{3}+k_{2}\right)\left(R_{0}-1\right)+a k_{2}\right)},
\end{aligned}
$$

where

$$
R^{*}=\frac{\left(r-d_{1}\right) a k_{2}+d_{3} d_{4}\left(d_{2}+k_{2}\right)}{d_{3} d_{4}\left(d_{2}+k_{2}\right)}
$$

\subsection{Positivity and boundedness of solutions}

For the problem deal with cell population evolution, the cell densities should remain non-negative and bounded. In this subsection, we will establish the positivity and boundedness of solutions of the model (1). First of all, for biological reasons, the parameters $x_{0}, y_{0}, s_{0}$ and $v_{0}$ must be larger than or equal to 0 . Hence, we have the following result

Proposition 21 The solutions of the problem (1) exist. Moreover, they are bounded and nonnegative for all $t>0$.

Proof Notice that system (1) is locally lipschitzian at $t=$ 0 . Hence the solution of this system exists and is unique on $[0, b)$ for some $b>0$. Observe that if $x(0)=0$, then $x \equiv 0$ for all $t>0$. Thus, we assume below that $x(0)>0$. We also have the following:

$\left.\dot{y}\right|_{y=0}=\frac{k_{1} x v}{x+v} \geq 0,\left.\dot{s}\right|_{s=0}=k_{2} y \geq 0$ and $\left.\dot{v}\right|_{v=0}=a s \geq 0$. This shows that $x(0)=x_{0}>0, y(0)=y_{0} \geq 0, s(0)=s_{0} \geq$ 0 and $v(0)=v_{0} \geq 0$ for all $t \in[0, b)$.

On the other hand, for the boundedness of the solutions, we have the following:

$$
\frac{d T(t)}{d t}=r x(t)\left(1-\frac{T(t)}{T_{m}}\right)-d_{1} x(t)-d_{2} y(t)-d_{3} s(t),
$$

since

$$
T(t) \leq T_{m} \text { and } x(t) \leq T(t),
$$

we obtain

$$
\frac{d T(t)}{d t} \leq r T(t)\left(1-\frac{T(t)}{T_{m}}\right) \leq r T(t)
$$

thus

$$
T(t) \leq T_{0} e^{-r t}
$$

with $T_{0}=x_{0}+y_{0}+s_{0}$.

We conclude that $T$ is bounded, which means also that $x, y$ and $s$ are bounded.

From the last equation of (1), we have

$$
v(t) \leq v(0) e^{-d_{4} t}+a \int_{0}^{t} s(t) e^{(\xi-t) d_{4}} d \xi,
$$

therefore

$$
v(t) \leq v(0)+\frac{a}{d_{4}}\|s\|_{\infty}\left(1-e^{-d_{4} t}\right) .
$$

Since $\left(1-e^{-d_{4} t}\right) \leq 1$, we conclude that $v$ is bounded.

\subsection{The stability analysis}

First, the jacobian matrix of the system (1) is given by

$$
J=\left(\begin{array}{ccrc}
r\left(1-\frac{2 x+y+s}{T_{m}}\right)-d_{1}-\frac{k_{1} v^{2}}{(x+v)^{2}} & -\frac{r x}{T_{m}} & -\frac{r x}{T_{m}} & -\frac{k_{1} x^{2}}{(x+v)^{2}} \\
\frac{k_{1} v^{2}}{(x+v)^{2}} & -\left(d_{2}+k_{2}\right) & 0 & \frac{k_{1} x^{2}}{(x+v)^{2}} \\
0 & k_{2} & -d_{3} & 0 \\
0 & 0 & a & -d_{4}
\end{array}\right)
$$

A straightforward calculation gives the following expression for the basic reproductive number in the model (1):

$$
R_{0}=\frac{a k_{1} k_{2}}{d_{3} d_{4}\left(d_{2}+k_{2}\right)} .
$$

\subsubsection{Stability of the infection-free equilibrium point $E_{f}$}

Here, we will analyze locally asymptotical stability and globally asymptotical stability of the disease-free equilibrium $E_{f}$.

Proposition 22 The free equilibrium point $E_{f}$ is locally asymptotically stable when $R_{0}<1$ and unstable when $R_{0}>$ 1.

Proof The Jacobian matrix at $E_{f}$ is given by

$$
J_{E_{f}}=\left(\begin{array}{ccrc}
-\left(r-d_{1}\right) & -\left(r-d_{1}\right) & -\left(r-d_{1}\right) & -k_{1} \\
0 & -\left(d_{2}+k_{2}\right) & 0 & k_{1} \\
0 & k_{2} & -d_{3} & 0 \\
0 & 0 & a & -d_{4}
\end{array}\right)
$$

The characteristic polynomial of $J_{E_{f}}$ is

$$
P_{E_{f}}(\xi)=\left(\xi+\left(r-d_{1}\right)\right)\left[\xi^{3}+a_{1} \xi^{2}+a_{2} \xi+a_{3}\right],
$$

where

$$
\begin{aligned}
& a_{1}=d_{2}+d_{3}+d_{4}+k_{2}, \\
& a_{2}=d_{3} d_{4}+\left(d_{3}+d_{4}\right)\left(d_{2}+k_{2}\right), \\
& a_{3}=d_{3} d_{4}\left(d_{2}+k_{2}\right)-a k_{1} k_{2} .
\end{aligned}
$$

While $\xi_{1}=-\left(r-d_{1}\right)$ is a negative eigenvalue, the other three eigenvalues are given by the solution of the following cubic equation,

$$
\xi^{3}+a_{1} \xi^{2}+a_{2} \xi+a_{3}=0 .
$$

It is clear that, $a_{1}>0, a_{2}>0$ and $a_{1} a_{2}-a_{3}=d_{3} d_{4}\left(d_{3}+d_{4}\right)+$ $\left(d_{2}+k_{2}\right)\left(d_{3}+d_{4}\right)\left(d_{2}+d_{3}+d_{4} k_{2}\right)+a k_{1} k_{2}>0$. If $R_{0}<1$, then $a_{3}>0$. From the Routh-Hurwitz Theorem given in [?], all roots of this equation have negative real parts. Then $E_{f}$ is locally asymptotically stable when $R_{0}<1$.

Proposition 23 The free equilibrium point $E_{f}$ is globally asymptotically stable when $R_{0}<1$, provided the initial data satisfies. 
Proof From positivity of solutions, we have that $s$ and $v$ satisfy the differential inequality

$$
\begin{aligned}
\frac{d s}{d t} & \leq k_{2} y(t)-d_{3} s(t), \\
\frac{d v}{d t} & \leq a s(t)-d_{4} v(t) .
\end{aligned}
$$

It is clear from (1) that if $(s(t), v(t)) \rightarrow(0,0)$ then $x(t) \rightarrow$ $T_{m}$ and $y(t) \rightarrow 0$. We can apply Theorem 3.2 of [10] and conclude that $(s(t), v(t)) \rightarrow(0,0)$ as $t \rightarrow \infty$ when $R_{0}<1$. Thus, $E_{f}$ is globally asymptotically stable.

\subsubsection{Stability of the endemic equilibrium point $E^{*}$}

In this part, we discuss the local stability of the endemic infection equilibrium point $E^{*}$.

Proposition 24 If $R^{*}<R_{0}$ or $R_{0}<1$, the point $E^{*}$ does not exist. The endemic equilibrium point $E^{*}$ is locally asymptotically stable when $R_{0}>1$ and $R^{*}>R_{0}$.

Proof From the expression of $E^{*}$ we observe that this point exists when $1 \leq R_{0} \leq R^{*}$ and it becomes $E_{f}$ when $R_{0}=1$. We assume that $1 \leq R_{0} \leq R^{*}$.

The Jacobian matrix at the endemic equilibrium point $E^{*}$ is given by

$J_{E^{*}}=\left(\begin{array}{ccrc}r\left(1-\frac{2 x^{*}+y^{*}+s^{*}}{T_{m}}\right)-d_{1}-\frac{k_{1} v^{* 2}}{\left(x^{*}+v^{*}\right)^{2}} & -\frac{r x^{*}}{T_{m}} & -\frac{r x^{*}}{T_{m}} & -\frac{k_{1} x^{* 2}}{\left(x^{*}+v^{*}\right)^{2}} \\ \frac{k_{1} v^{* 2}}{\left(x^{*}+v^{*}\right)^{2}} & -\left(d_{2}+k_{2}\right) & 0 & \frac{k_{1} x^{* 2}}{\left(x^{*}+v^{*}\right)^{2}} \\ 0 & k_{2} & -d_{3} & 0 \\ 0 & 0 & a & -d_{4}\end{array}\right)$

The characteristic polynomial of $J_{E^{*}}$ is

$$
P_{E^{*}}(\xi)=\xi^{4}+b_{1} \xi^{3}+b_{2} \xi^{2}+b_{3} \xi+b_{4},
$$

From the Routh-Hurwitz theorem applied to the fourth order polynomial, the eigenvalues of the jacobian matrix (3) have negative real parts since we have $b_{1} b_{2}>b_{3}$ and $b_{1} b_{2} b_{3}>b_{3}^{2}+b_{1}^{2} b_{4}$. Consequently, we obtain the asymptotic local stability of the endemic point $E^{*}$.

\section{Numerical simulations}

In order to carried out the numerical simulations, we have used the Euler finite-difference scheme method. The parameter values or ranges used are presented in Table 1.

Figure 1, shows the behavior of the infection during the first 800 days of observation. We clearly see that the curves converge to the disease-free steady state $E_{f}=(805,0,0,0)$. In this case the basic reproduction number is less than unity $\left(R_{0}=3.597 \times 10^{-3}<1\right)$ which supports our theoretical result about the stability of $E_{f}$. Figure 2, shows the evolution of the infection during the first 800 days of observation. We use a same set of parameter values as those in Table 1, but we vary the value of $r$. For $r=1$ all the curves converge to the endemic equilibrium $E^{*}=(443.237,152.462,290.353$, $1675.439), R_{0}=4.78>1$ and $R^{*}=10.427$ which prove the stability of this endemic equilibrium. For $r=2$ all the curves also converge to the endemic equilibrium $E^{*}=$
Table 1. Parameters, their symbols and default values used in HIV literature.

\begin{tabular}{lll}
\hline Parameters & Value & References \\
\hline$r$ & $0.03-3$ day $^{-1}$ & {$[7]$} \\
$T_{m}$ & $1500 \mathrm{~mm}^{-3}$ & {$[7]$} \\
$d_{1}$ & 0.0139 days $^{-1}$ & {$[9]$} \\
$k_{1}$ & $2.5 \times 10^{-4}-0.5 \mathrm{~mm}^{-3}$ day $^{-1}$ & {$[9]$} \\
$d_{2}$ & 0.0495 days $^{-1}$ & {$[9]$} \\
$k_{2}$ & 1.1 days $^{-1}$ & {$[9]$} \\
$d_{3}$ & 0.5776 days $^{-1}$ & {$[9]$} \\
$a$ & $2-1250$ virion cell $^{-1}$ & {$[9]$} \\
$d_{4}$ & $0.3466-2.4$ day $^{-1}$ & {$[9]$} \\
\hline
\end{tabular}
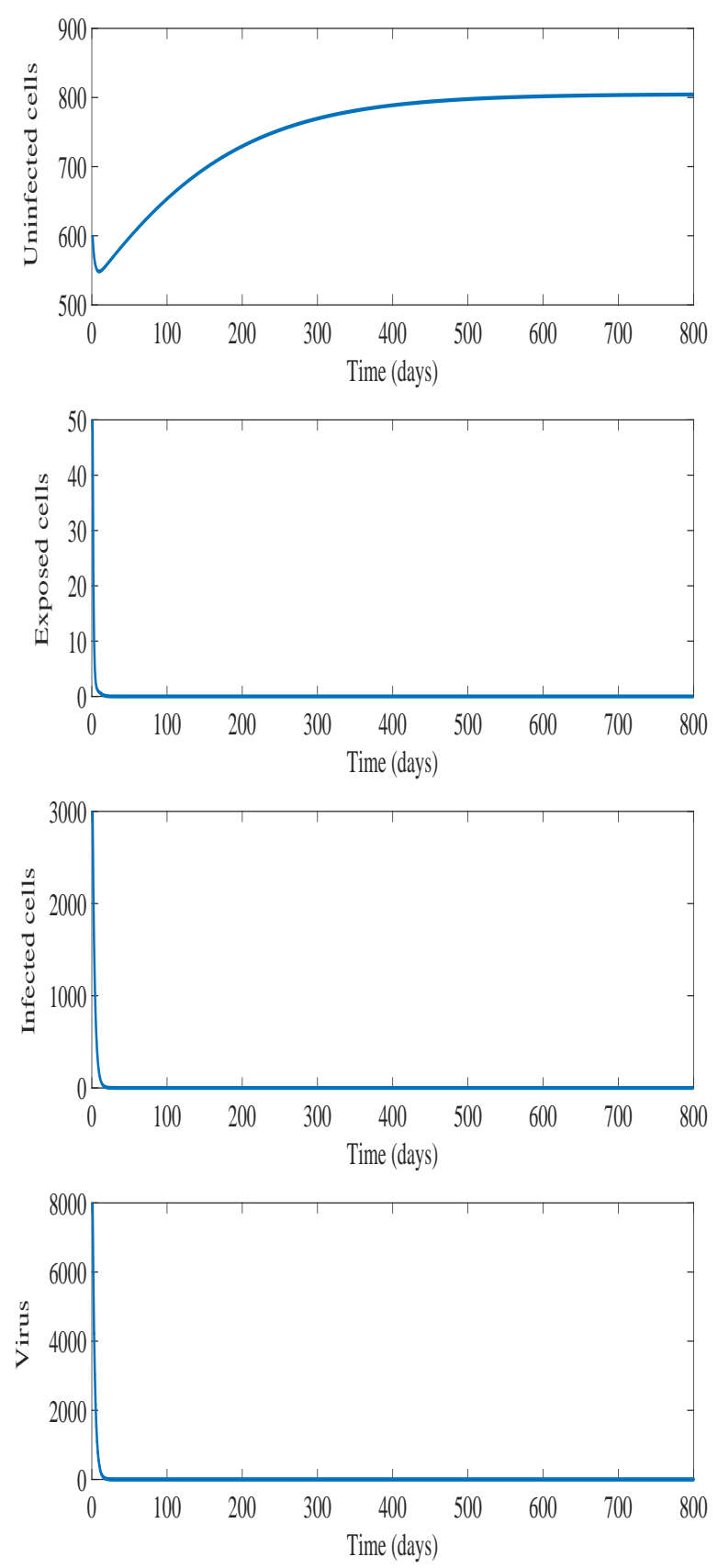

Fig. 1. Behavior of the infection dynamics for $r=0.03, T_{m}=$ $1500, k_{1}=0.0027, d_{1}=0.0139, d_{2}=0.0495, k_{2}=1.1, d_{3}=$ $0.5776, a=5$ and $d_{4}=2.4$, which correspond to the stability of the free-equilibrium point $E_{f}$. 

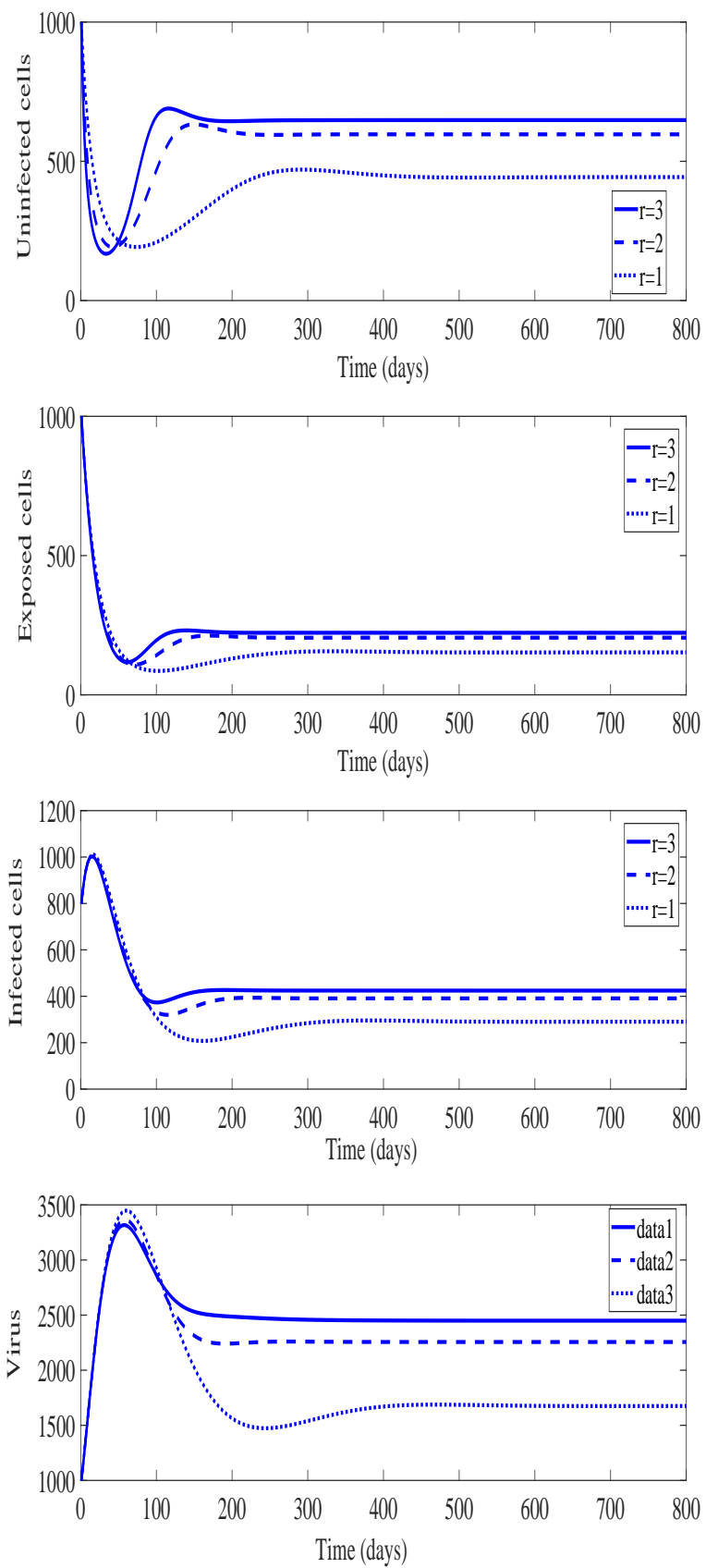

Fig. 2. Behavior of the infection dynamics for $T_{m}=1500, k_{1}=$ $0.5, d_{1}=0.0139, d_{2}=0.0495, k_{2}=1.1, d_{3}=0.5776, a=2$ and $d_{4}=0.3466$ which correspond to the stability of the endemicequilibrium point.

$(596.797,205.282,390.946,2255.895)$ which prove the stability of this endemic equilibrium, here $R_{0}=4.78>1$ and $R^{*}=19.987$. For $r=3$ all the curves converge also to the endemic equilibrium $E^{*}=(647.984,222.889,424.477$, $2449.380), R_{0}=4.78>1$ and $R^{*}=29.547$ which prove the stability of this endemic equilibrium. For all cases $R_{0}>$ 1 and $R^{*}>R_{0}$, the numerical simulations agree with our theoretical finding concerning the stability of the free and endemic equilibria. With low value of $r$, a significant reduce of infected cells in latent stage, productively infected cells and the viral lowed is observed. One can conclude that the logistic growth of the uninfected cells plays an essential role in the HIV viral dynamics.

\section{Conclusion}

In this work, we have studied a mathematical model of HIV dynamics with logistic function that describes the interactions between HIV and $\mathrm{CD}^{+}{ }^{+} \mathrm{T}$ cells. The considered model includes four differential equations describing the interaction between the uninfected $\mathrm{CD} 4^{+} \mathrm{T}$ cells, infected $\mathrm{CD}^{+} \mathrm{T}$ cells in latent stage, productively infected $\mathrm{CD} 4^{+}$ $\mathrm{T}$ cells and free virus (HIV). First, the existence, positivity and boundedness of solutions are proved. Next, both the local stability and global stability of the disease-free equilibrium and local stability of the endemic equilibria are established. Some numerical simulations are performed in order to confirm the theoretical results concerning the equilibrium stability.

\section{References}

1. Wordld Health Organization HIV/AIDS facts, http://www.who.int/mediacentre/factsheets/ fs360/en/index.html, accessed July (2017).

2. M.A. Chesney, J. ICKOVICS, F.M. HECHT, G. Sikipa, J. Rabkin, Adherence: a necessity for successful HIV combination therapy, AIDS (London, England). 13 (1999) 271-8.

3. S. Pankavich, The Effects of Latent Infection on the Dynamics of HIV, Differential Equations and Dynamical Systems. 24 (2016) 281-303.

4. L. Rong, M.A. Gilchrist, Z. Feng, A.S. Perelson, Modeling within-host HIV1 dynamics and the evolution of drug resistance: tradeoffs between viral enzyme function and drug susceptibility, Journal of Theoretical Biology. 247 (2007) 804-818.

5. L. Rong, A.S. Perelson, Asymmetric division of activated latently infected cells may explain the decay kinetics of the HIV-1 latent reservoir and intermittent viral blips, Mathematical biociences. 217 (2009) 7787.

6. B. Buonomo, C. Vargas-De-Leon, Global stability for an HIV-1 infection model including an eclipse stage of infected cells, Journal of Mathematical Analysis and Applications. 285 (2012) 709-720.

7. Y. Wang, Y. Zhou, J. Wu, J. Heffernan, Oscillatory viral dyanamics in a delayed HIV pathogenesis model, Mathematical biociences. 209 (2009) 104-112.

8. L. Wang, M.Y. Li, Mathematical analysis of the global dynamics of a model for HIV infection of $\mathrm{CD}^{+} \mathrm{T}$ cells, Mathematical biociences. 200 (2006) 44-57.

9. Q. Sun, L. Min, Y. Kuang, Global stability of infection-free state and endemic infection state of a modified human immunodeficiency virus infection model, IST systems biology. 9 (2015) 95-103.

10. S. A. Gourley, Y. Kuang and J. D. Nagy, Dynamics of a delay differential equation model of hepatitis B virus infection, Journal of Biological Dynamics. 2 (2008) 140-153. 